\title{
Fuzzy modelling of knee joint with genetic optimization
}

\author{
B.S.K.K. Ibrahim*, M.O. Tokhi, M.S. Huq, R. Jailani and S.C. Gharooni \\ Department of Automatic Control and System Engineering, University of Sheffield, Sheffield, United Kingdom
}

\begin{abstract}
Modelling of joint properties of lower limbs in people with spinal cord injury is significantly challenging for researchers due to the complexity of the system. The objective of this study is to develop a knee joint model capable of relating electrical parameters to dynamic joint torque as well as knee angle for functional electrical stimulation application. The joint model consists of a segmental dynamic, time-invariant passive properties and uncertain time-variant active properties. The knee joint model structure comprising optimised equations of motion and fuzzy models to represent the passive viscoelasticity and active muscle properties is formulated. The model thus formulated is optimised using genetic optimization, and validated against experimental data. The developed model can be used for simulation of joint movements as well as for control development. The results show that the model developed gives an accurate dynamic characterisation of the knee joint.
\end{abstract}

Keywords: Knee joint model, viscoelasticity, functional electrical stimulation, fuzzy model, genetic algorithm

\section{Introduction}

Functional electrical stimulation (FES) is a promising way to restore mobility to individuals paralyzed due to spinal cord injury (SCI). Musculoskeletal modelling provides researchers with great potential for studying the biomechanics of movement through simulation, which would otherwise require resorting to invasive methods [22]. Modelling, and hence simulation study can greatly facilitate to test and tune various FES controllers. In order to develop a control strategy for the FES to move the leg correctly, an accurate model of the stimulated muscle has to be used. Accurate models can facilitate the design of stimulation patterns and control strategies that will produce the desired force and motion [24].

\footnotetext{
*Corresponding author: Babul Salam Ksm Kader Ibrahim. E-mail: babulsalam@yahoo.com, babul@uthm.edu.my.
}

Modelling the human joints is difficult due to the combination of complex structural anatomy, complicated movement and dynamics and often indeterminate muscle function [2]. Moreover, most models built either on experimental or physiological bases are not appropriate for FES control applications [23]. One way to develop this model for FES control applications is to use mathematical approaches. As a result, many researchers have developed mathematical model of electrically stimulated muscles based on Hill-type $[10,15]$, Huxley-type [11], analytical approaches [14, 17] and physiology approach [26]. Accurate models of artificial muscle activation in healthy or paraplegic subjects have been developed but the complexities of the system resulting mathematical representation have a large number of parameters that make the model identification process difficult.

Fuzzy logic has long been known for its ability to handle a complex nonlinear system without a math- 
ematical model. Fuzzy logic is the fastest growing soft computing tool in medicine and biomedical engineering [3] with its major strength in nonlinear output mapping [28]. Biological inspired computation techniques such as genetic algorithms (GA) are used in designing fuzzy models, particularly for generating fuzzy rules and adjusting membership functions. To the authors' knowledge, there is no extensive study in the literature regarding musculoskeletal modelling using fuzzy evolutionary systems.

Knee joint model consists of active muscle properties and passive viscoelasticity and the equation of motion of the lower limb. Active properties include quadriceps muscle activation and muscle contraction while passive viscoelasticity includes passive viscous moment and elastic moment of knee joint. Equations of motion are applied to compute joint movements from joint moments taking into account gravitational and inertial effects. The active joint moment is added with the passive joint moment as an input (torque) to the lower limb model and this will produce the knee angle as the output.

In this paper, a new knee joint model including optimised segmental dynamics and optimised dynamic characterization of passive and active properties based on experimental data is proposed. The model of a dynamic system of the lower limb is derived using well known Kane's equations [29]. The anthropometric inertial parameters such as foot mass, shank mass, moment of inertia about centre of mass (COM) and position of COM along the segmental length of the limb in these equations are optimised using genetic algorithm (GA). GA adjusted fuzzy models are used to represent the passive viscoelasticity (combination of elastic and viscous moments) of knee joint and the active properties (combination of the muscle activation and contraction) of the quadriceps muscle.

The aim of the present study is to develop a model and procedure, with due consideration of the above constraints, that would enable to identify for a person the relationship between the stimulation pulsewidth and the motion produced by the stimulated muscles. A generic methodology is presented that can be adopted for accurate estimation of anthropometric inertia parameters and model of passive viscoelasticity and active properties of knee joint specific to individuals with SCI. The same approach could also be used, in principle, for different persons (e.g. with different ages, weights, heights, and gender).

\section{Materials and method}

\subsection{Knee joint moment equation}

The shank-quadriceps dynamics are modelled as the interconnection of passive and active properties of muscle model and the segmental dynamics. The total knee-joint moment is given by [17]:

$$
M_{\mathrm{i}}=M_{\mathrm{a}}+M_{\mathrm{g}}+M_{\mathrm{s}}+M_{\mathrm{d}}
$$

where $M_{\mathrm{a}}$ refers to an active knee joint moment produced by electrical stimulation, $M_{\mathrm{S}}$ is the knee joint elastic moment and $M_{\mathrm{d}}$ is the viscous moment representing the passive behaviour of the knee joint. The $M_{\mathrm{i}}$ and $M_{\mathrm{g}}$ are represented by equation of motion for dynamic model of the lower limb. The $M_{\mathrm{S}}$ and $M_{\mathrm{d}}$ are combined and represented by a fuzzy model as passive viscoelasticity. Figure 1 shows a schematic

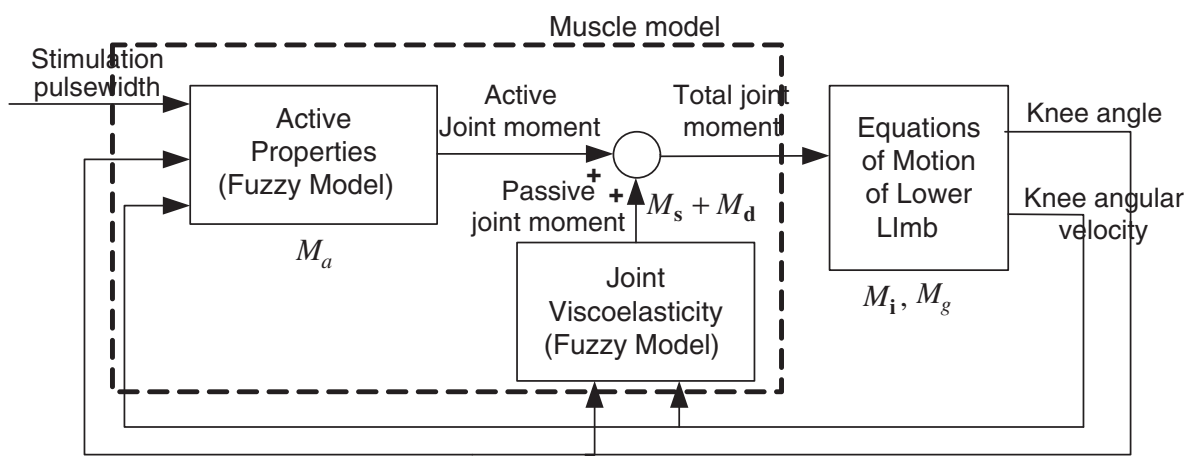

Fig. 1. Schematic representation of the knee joint model. 
representation of the knee joint model consisting of active properties, passive viscoelasticity and lower limb model.

The subject participating in this work was a 48 yearold T2\&T3 incomplete paraplegic male with 20 years post-injury with height $=173 \mathrm{~cm}$ and weight $=80 \mathrm{~kg}$. Informed consent was obtained from the subject.

\subsection{Optimization and estimation procedure}

In this work, the optimisation process is divided into two stages; first GA is used to estimate the anthropometric inertia parameter of lower limb and to adjust the fuzzy parameters to represent the non-linear viscoelasticity by minimizing the error between the data obtained from pendulum test and the model prediction. Then these optimized equations of motion and fuzzy viscoelasticity model are used in modelling the active properties. In the second stage, the active properties are modelled based on input and output data from electrically stimulated test using multi-objective genetic algorithm (MOGA) with two objectives; to minimize the prediction error to fit the experimental data.

\subsection{Equations of motion for dynamic model of the lower limb}

A schematic diagram of the lower limb model is shown in Fig. 2, where $q_{2}=$ shank length, $r_{1}=$ position of COM along the shank, $r_{2}=$ position of COM along the foot, $\theta_{1}=$ knee angle and $\theta_{2}=$ ankle angle. The complexity in analysing multi-joint structure is often reduced through reducing the number of degrees of freedom (DoF) to a manageable level [9]. In this research, the foot at neutral position is defined as $0^{\circ}$. Hence, the motion dynamics can be represented in a simpler form. The gravitational $\left(M_{\mathrm{g}}\right)$ moment is represented by:

$$
M_{g}=m_{1} g \cos \theta_{1} r_{1}+m_{2} g \cos \theta_{1} q_{2}
$$

The inertial $\left(M_{\mathrm{i}}\right)$ moment is represented by mathematical model of the lower limb based on Kane's equations as follows:

$$
M_{\mathrm{i}}=-m_{2} q_{2} \dot{\theta}_{1}^{2} r_{2}-I_{1} \ddot{\theta}_{1}-m_{1} r_{1}^{2} \ddot{\theta}_{1}-m_{2} q_{2}^{2} \ddot{\theta}_{1}
$$

where, $m_{1}=$ shank mass, $m_{2}=$ foot mass, $I_{1}=$ moment of inertia about COM, $\dot{\theta}_{1}=$ knee velocity, $\ddot{\theta}_{1}=$ knee acceleration, $g=$ gravity. Anthropometric measure-

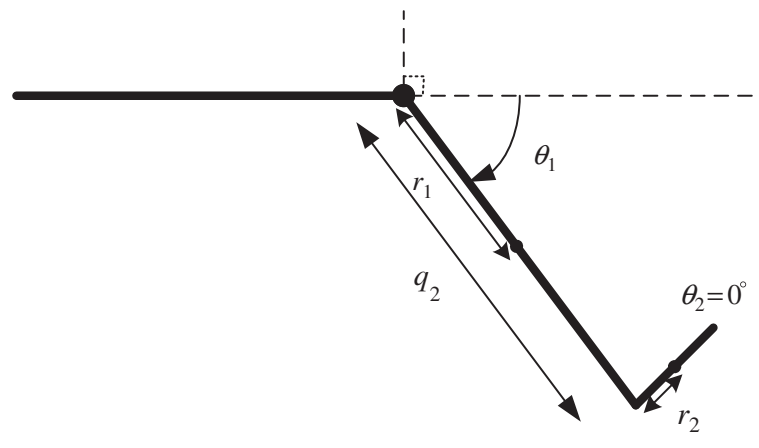

Fig. 2. Lower limb model.

Table 1

Anthropometric data of subject

\begin{tabular}{lc}
\hline Segment & Length $(\mathrm{m})$ \\
\hline Shank length & 0.426 \\
Foot length & 0.168 \\
Approximated position of COM of shank & 0.213 \\
Approximated position of COM of soot & 0.084 \\
\hline
\end{tabular}

ments of length of the lower limb were made and these are shown in Table 1.

\subsection{Experimental protocol}

The experimental protocol entailed two phases as considered by Ferrarin and Pedotti [17]. The first involved a passive pendulum test of the lower limb to estimate the viscoelasticity of knee joint. The second phase implied stimulating the quadriceps muscle, recording the artificially induced movement and computing the produced active knee torque.

\subsubsection{Pendulum test}

Pendulum test can be used to evaluate passive elements such as viscosity and elasticity moments of the knee [27]. Viscoelasticity is combination of elasticity and viscosity and represents passive resistances to joint motion associated with the structural elements of the joint tissue and of the muscular-tendon complex [25]. The subject sat on a chair with arm rests, which allowed the lower leg to swing freely without knee flexion movement constrained by the sitting surface.

Movements of the ankle and hip joint were not taken into consideration to reduce the number of DoF and made solely dependent upon the knee joint position. For this reason during the experiments the ankle and hip joint of subject were fixed. The ankle angle was 
fixed at $0^{\circ}$ using Velcro. The hip angle was fixed at approximately $100^{\circ}$ from the horizontal using straps, so that hip extension was not possible. Thigh was supposed to be stationary and fixed using strap on the chair so that only the shank-foot dynamics were considered in the model. This was also to ensure that the limbs were within the sagittal plane.

Reflexive or voluntary activation of muscles acting on the knee during the pendulum test was monitored through the Electromyography (EMG) technique and repetition of the experiment to rule out uncertainty due to influence of pendulum movements. Continuous EMG technique was used for evaluating and recording the electrical activity produced by skeletal muscles [16] while doing the pendulum test [21]. EMG signal was recorded via bipolar surface electrodes on the main muscles on the knee joint (rectus femoris, vasti and hamstring muscles) to rule out such spastic limb motion during the passive pendulum trials as in [17].

The knee was slowly extended to a level close to the horizontal but where the subject still felt comfortable, by having the experimenter lift it with minimal acceleration. The leg was held in this position until the subject was fully relaxed and the EMG signals had become stable at almost zero level. Then the knee was released and permitting it to swing freely. The knee angle was recorded using a Biometric electro-goniometer with sampling frequency of $20 \mathrm{~Hz}$.

\subsubsection{Electrically stimulated quadriceps test}

Electrically stimulated quadriceps test was used to obtain the input and output data to model the active properties produced by FES. Active properties are the combination of muscle activation and contraction. Activation dynamics provide muscle response due to electrical stimulation. The contraction dynamics are the mechanical contraction and relaxation of muscle and tendon including their length and velocitydependent properties.

In this test, the subject sat in the same position as in the pendulum test. Two surface moistened $(2$ " $\times 3.5$ ") PALS neurostimulation self-adhesive electrodes were placed on the quadriceps muscle. The cathode (negative) placed just proximally over the estimated motor point of rectus femoris and the anode (positive) approximately $4-\mathrm{cm}$ proximal of the patella. HASOMED current-controlled stimulation device was used to send the signals to the muscle. The stimulation current was fixed to $40 \mathrm{~mA}$ and the frequency was set to $25 \mathrm{~Hz}$ with a doublet type pulse. The input (stimulation pulse- width) and the resultant output (knee angle) from this experiment are shown in Fig. 3.

Additional pendulum and electrical stimulation trials were recorded for the evaluation of the prediction capability of the models. These data have not been used in the model identification phase.

\subsection{Fuzzy modelling approach}

Modelling based on conventional mathematical tools is not well suited for dealing with complex and nonlinear systems. By contrast, a fuzzy inference system employing fuzzy 'if-then' rules can model the qualitative aspects of human knowledge and reasoning processes without employing precise quantitative analyses [7]. Fuzzy models are well suited for modelling non-linear systems such as passive viscoelasticity and active properties of the quadriceps muscle.

One of the major problems in the not so widespread use of fuzzy logic is the difficulty in designing membership functions (MFs) to suit a given problem. A systematic procedure for choosing the vector of parameters that specify the MF is still not available [22]. Tuning of the parameters by trial and error is often necessary for a satisfactory performance to be achieved. However, the optimisation of fuzzy parameters has been restricted due to large number of optimisation parameters. GA is most appropriate for non-linear models where location of the global optimum is a difficult task [18]. In this research, GA is used to tune a Mamdani-type fuzzy model due to its intuitive and interpretable nature of the rule base.

\subsection{Genetic algorithm}

Evolutionary computing, GAs are globally searching techniques, which are more likely to converge to the global optimum and emulate natural genetic operators such as selection, crossover, and mutation [8]. This evolutionary algorithm in conjunction with fuzzy logic has been used successfully in biomedical engineering in various applications [13]. The GA approach is able to search many points simultaneously as well as able to avoid local optima that traditional gradient descent algorithms might get stuck in [20]. It may be possible to use GA techniques to consider problems which may not be modelled as accurately using other approaches. Therefore, GA appears to be a potentially useful approach for these modelling purposes. 

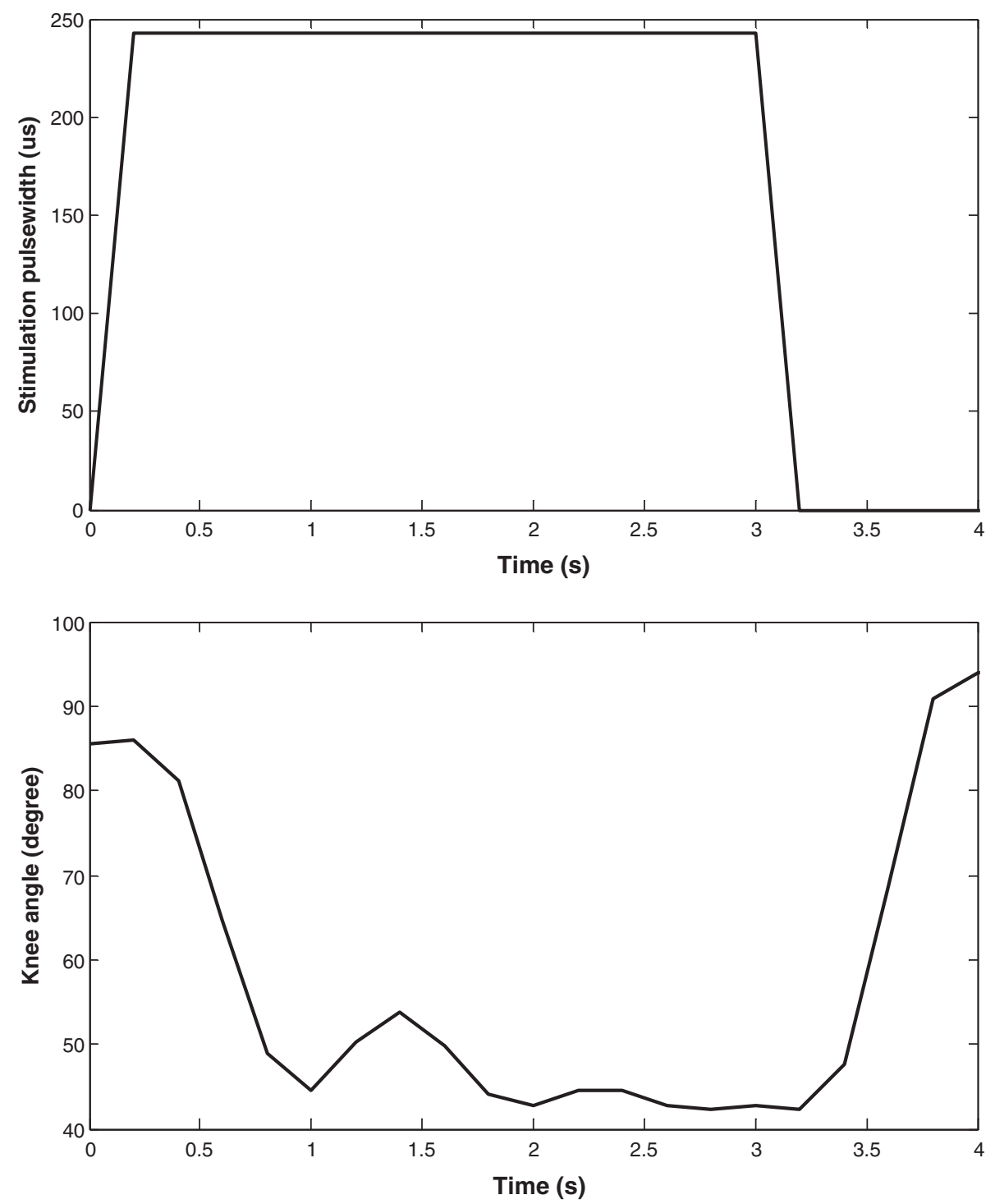

Fig. 3. Stimulation pulse-width (input) and the knee angle (output).

\subsection{Optimization of anthropometric inertia parameters and fuzzy viscoelasticity model using $G A$}

GA is used to optimize the fuzzy systems to represent the dynamic behaviour of passive viscoelasticity of the knee joint using fuzzy model and estimation of the anthropometric inertia parameters such as foot mass, shank mass, moment of inertia about COM and positions of COM along the segmental length of the lower limb as shown in Fig. 4. The ranges of optimisation values (minimum and maximum) of these parameters are necessary to ensure that the GA addresses the identification separately. Moreover, the repetition of this optimisation helps to ensure consistency of the identification. The goal of GA optimization process is to minimize the error between the knee angle obtained experimentally and from the model. The output prediction error is defined as:

$$
e(t)=y(t)-\hat{y}(t)
$$

where $y(t)$ is the experimental data and $\hat{y}(t)$ is the estimated current output of knee angle. The 'goodness of 


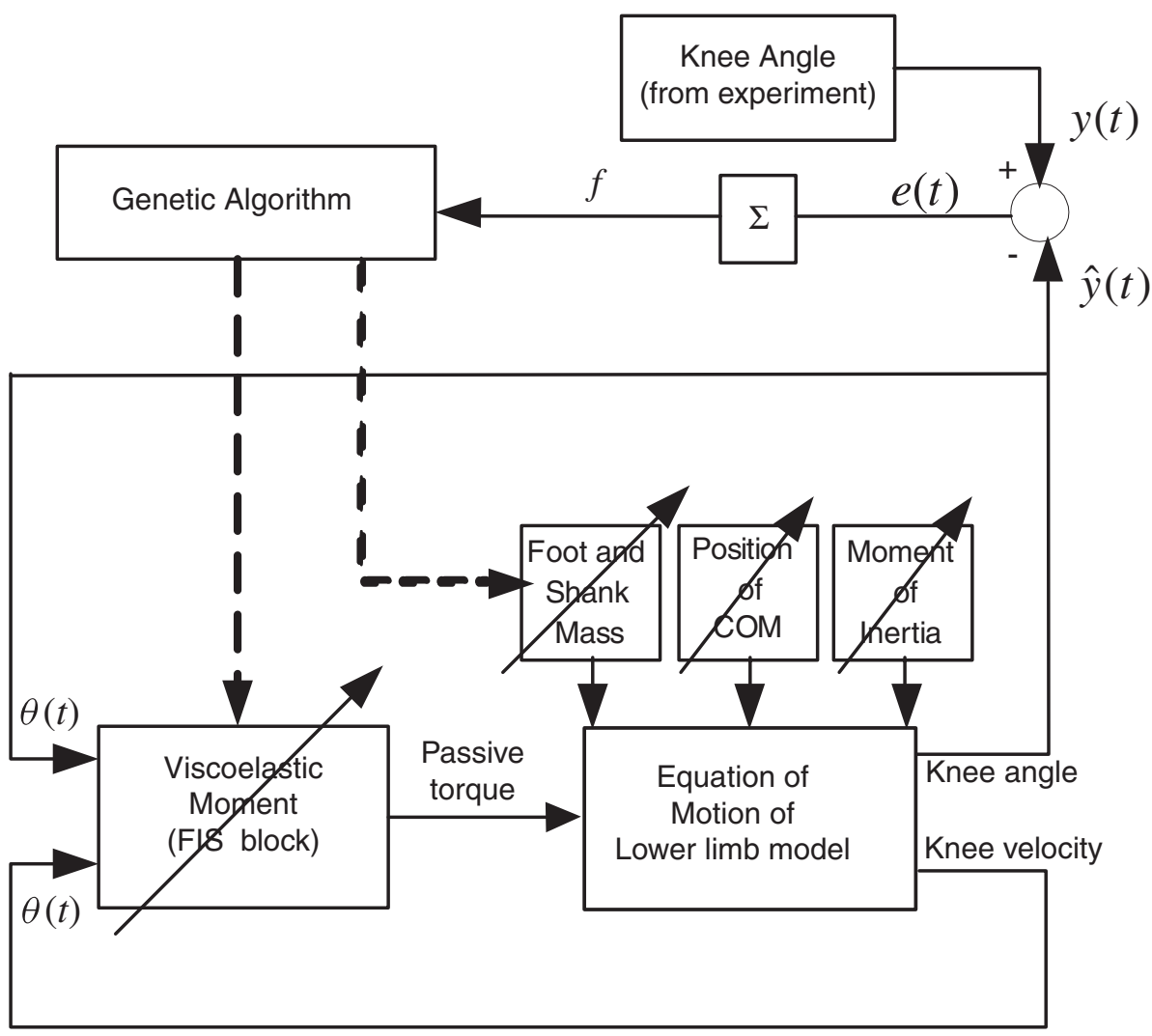

Fig. 4. Optimization of passive viscoelasticity and anthropometric inertia parameters.

fit' of the identified model is determined using the objective function by minimizing the mean-squared error (MSE):

$$
f_{1}=\left\{\frac{\sum_{i=1}^{N}(y(t)-\hat{y}(t))^{2}}{N}\right\}
$$

where $N$ is the number of sample values.

\subsubsection{Estimation of anthropometric inertia parameters}

The lower limb dynamics are complex and not well defined. The complexity of the leg inertial characteristics is not only due to the limb shape, i.e. different tissues (Lebiedowska, 2008). Indeed, even the location of the COM of elemental parts is imprecise, and comprehensive analyses of joint kinematics are extremely difficult. To quantify limb dynamics, accurate estimates of anthropometric inertia parameters (mass, location of COM, and moments of inertia) are needed.
2.7.1.1. Moments of inertia. Moments of inertia are fundamental parameters describing the mass distribution of body segments which enter into all computations involving segmental rotations. There is no unique way of calculating the moment of inertia. To obtain more accurate moment of inertia of the shank + ankle segment, the minimum $\left(0.32 \mathrm{~kg} \mathrm{~m}^{2}\right)$ and maximum $\left(0.58 \mathrm{~kg} \mathrm{~m}^{2}\right)$ range as reported by Lebiedowska (2003) is setup and GA optimization used to determine optimized value of moment of inertia within this range.

2.7.1.2. Mass and position of COM of shank and foot. Vatnsdal et al. [4] addressed the masses and positions of the COM of the lower limb as possible error sources in the lower limb model. Zatsiorsky and Seluyanov [30] estimated the mass on the basis of regression equations based on statistics measured from 100 young men using gamma-scanner method. However, this method has a standard error and it is hypothesized that paraplegics would have different measurements 
as compared with able-bodied subjects. Therefore, it can potentially introduce large errors, especially while modelling the passive elements, as the pendulum test results may be affected by those parameters.

Thus, fine tuning these parameters around the predicted values with an efficient stochastic search algorithm such as GA could lead to good solution with immense potential of extracting accurate subject specific results. GA is used to optimise the foot and shank mass within this range as shown in Table 2, while the approximated positions of COM along the segment length are assumed as in the centre of the segment length. To obtain accurate positions, the position of the COM of shank and foot are optimised with $\pm 10 \%$ tolerance.

\subsubsection{Optimization of fuzzy model as passive viscoelasticity}

The Mamdani type fuzzy inference with triangular membership functions is used to represent passive viscoealsticity as a non-linear function of knee angle and knee angular velocity. Piecewise linear triangular membership functions are preferred, because of their simplicity and efficiency with respect to computability. An automatic fuzzy system design method integrates three stages; determines membership functions, the rule-consequent parameters, and scaling factor at the same time. There are 73 parameters for 25 rules that are optimized. A breakdown of optimized parameter of the fuzzy system is as follows:-

i) 45 parameters relating to the 15 triangular MFs (3-element vector that determines the break points for each MF),

ii) 25 weights associated with the fuzzy rules,

iii) 3 scaling factors for the normalization and denormalization of 2 inputs and 1 output of fuzzy model respectively.

The weights of the 25 fuzzy rules are optimised between 0 and 1 with 2 decimal points in order to get reasonable accuracy. The rules are identified based on practical experience with reference to relationship between knee angle and velocity and passive torque. These identified rules help to reduce the computational time of the optimisation process. Table 3 summarizes the rule base for the fuzzy model encompassing possible AND combinations of the input fuzzy values.
Table 2

Segment mass of subject

\begin{tabular}{lccc}
\hline $\begin{array}{l}\text { Segment } \\
\text { mass }\end{array}$ & $\begin{array}{c}\text { Zatsiorsky and } \\
\text { Seluyanov's method }\end{array}$ & $\begin{array}{c}\text { Standard } \\
\text { error }\end{array}$ & $\begin{array}{c}\text { Range } \\
(\mathrm{kg})\end{array}$ \\
\hline Foot & $1.06 \mathrm{~kg}$ & $0.11 \mathrm{~kg}$ & $0.95-1.17$ \\
Shank & $3.42 \mathrm{~kg}$ & $0.23 \mathrm{~kg}$ & $3.19-3.65$ \\
\hline
\end{tabular}

Table 3

Rule base of the fuzzy model

\begin{tabular}{llllll}
\hline Knee angle & \multicolumn{5}{c}{ Knee angular velocity } \\
\cline { 2 - 6 } & NB & NS & ZO & PS & PB \\
\hline NB & PB & PB & PB & PS & ZO \\
NS & PB & PB & PS & ZO & NS \\
ZO & PB & PS & ZO & NS & NB \\
PS & PS & ZO & NS & NB & NB \\
PB & ZO & NS & NB & NB & NB \\
\hline
\end{tabular}

$\mathrm{NB}=$ Negative big, $\mathrm{NS}=$ Negative small, $Z=$ Zero $P S=$ Positive small, $\mathrm{PB}=$ Positive big .

\subsection{Optimisation of fuzzy model as active properties using MOGA}

There is often a problem in this case caused by the large number of rules which depend on the input data and number of MF [12]. This usually leads to a significant increase in the qualitative complexity in terms of poor transparency and unclear interpretation of the fuzzy rules as well as quantitative complexity in terms of increased number of operations during the fuzzy inference process [31]. Therefore, successful fuzzy models should take into account the number of rules with high efficiency. This is a multi-objective optimization problem.

Thus, MOGA is used to minimize the prediction error and the weighting factors of the fuzzy rules simultaneously. Fitness sharing technique as proposed by Fonseca and Fleming [5] is applied. Optimization of active properties using MOGA with integrating estimated passive joint viscoelasticity and optimized equation of motion is shown in Fig. 5. The automatic optimization is implemented in MATLAB with MOGA Toolbox with two-point crossover and mutation operators to optimise these parameters. Population size was set to 50 and crossover and mutation probabilities were 0.8 and 0.001 respectively.

Because these design stages may not be independent, it is important to consider them simultaneously to find the optimal solution. The first objective of MOGA optimization process is to minimize the error between the knee angle obtained experimentally and from the 


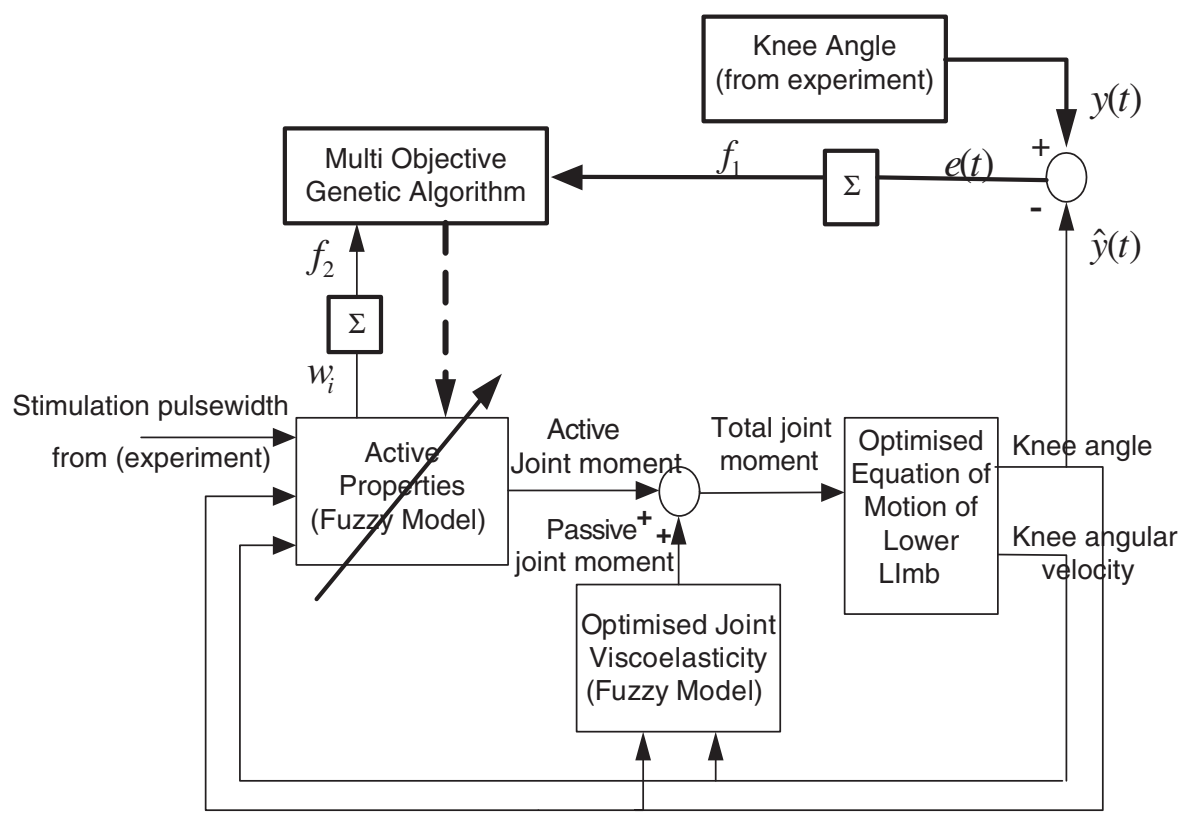

Fig. 5. Optimization of active properties.

model as equation (4). The second objective of MOGA is to minimize the sum of the weighting factor of the fuzzy rules without compromising the first objective:

$$
f_{2}=\sum_{i=1}^{n} w_{i}
$$

where $w_{i}$ is the $i$ 'th rule with $n$ as the total number of rules.

This minimization process compresses a fuzzy system with large number of rules into a smaller fuzzy system by removing the inactive fuzzy rules and redundancy in the fuzzy rule base. As a result of this compression, the number of operations during the fuzzy inference process is significantly reduced without compromising the quality of the solution by reducing the complexity of the fuzzy model. Following minimization of the weighting factors of the fuzzy rules, the average weighting factor is calculated as:

$$
w_{\text {ave }}=\frac{\sum_{i=1}^{N} w_{i}}{N}
$$

Then the rules with weighting factors smaller than the average weighting factor value are deleted. Finally this will end up with reduced number of rules to minimize the fuzzy model complexity.
Since the exact information about relationship between inputs and output are unknown, there are 439 parameters for 125 rules that are optimized. A breakdown of optimized parameters of the fuzzy system is as follows:

i) 60 parameters relating to the 20 triangular MFs, (3-element vector that determines the break points for each MF).

ii) $125 \mathrm{MFs}$ between Negative Big, Negative Small, Zero, Positive Small and Positive Big for all rules,

iii) 125 fuzzy operators for the rule's antecedent between OR and AND for all rules,

iv) 125 weights that are to be applied to the rule between 0 and 1 for all rules.

v) 4 scaling factors for the normalization and denormalization.

The first rule for this model with 3 inputs (stimulation pulsewidth, knee angle, knee angular velocity) and an output (active joint moment) is as: 'If input 1 is MF1 and/or input 2 is MF1 and/or input 3 is MF1 then output 1 is between MF1 to MF5 with weight between 0 and 1 '. Since there are 3 inputs with 5 MFs, the combination of different rules for this system can reach up to 125 rules. 


\section{Results}

\subsection{Passive viscoleasticity and anthropometric inertia parameters}

The population size of GA was set to 50 and crossover and mutation probabilities were 0.8 and 0.001 respectively. The automatic GA optimization process was set to generate up to 200 generations of solutions. The best solution was kept and the rest were discarded until there was no significant change in the MSE observed after the 165th generation. The minimum MSE achieved was 1.87. The response of the model was tested and the result is shown in Fig. 6. The results showed that the model parameters were estimated well and that the fit between the model and the experimental data was good.

The optimized values of the anthropometric inertia parameters such as masses, moment of inertia about COM and positions of COM along the segmental length of the subject's lower limb are shown in Table 4. The accuracy of these optimised anthropometric inertia parameters was verified through repeated process of optimisation.

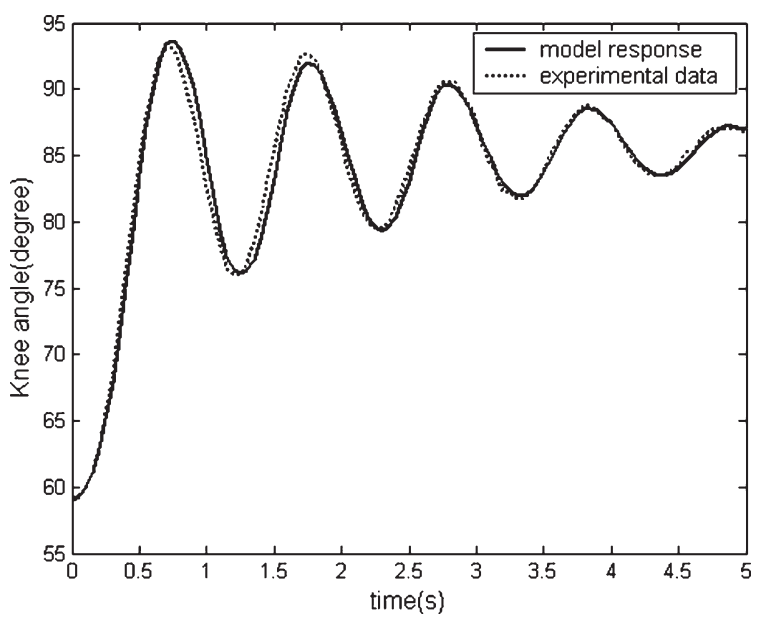

Fig. 6. Responses of the pendulum test and the model.

Table 4

Optimised value of anthropometric inertia parameters

\begin{tabular}{lc}
\hline Parameter & Optimised value \\
\hline Foot mass & $0.95 \mathrm{~kg}$ \\
Shank mass & $3.5 \mathrm{~kg}$ \\
Moment of inertia & $0.36 \mathrm{~kg} \mathrm{~m}^{2}$ \\
Position of COM of foot & $0.085 \mathrm{~m}$ \\
Position of COM of shank & $0.22 \mathrm{~m}$ \\
\hline
\end{tabular}

The vicoelasticity is represented by a fuzzy model The GA optimization was used to optimise 73 parameters including 45 associated with the triangular membership functions, 25 weights associated with the fuzzy rules and 3 scaling factors of fuzzy model. The resultant fuzzy rules are shown in Table 5, where the values in the bracket indicate the weighting factor for the corresponding rule fired. The scaling factor for the normalization and de-normalization of two inputs and output were $0.009,0.005$ and 35 respectively. Fig. 7 shows the optimally shaped inputs and output MFs of passive viscoelascity model.

Model validation is possibly the most important step in the modelling process. The model and the optimised parameters obtained from the optimization process were validated in terms of consistency and the prediction error. Two validation tests were conducted; first the model was validated with a set of data different from the training data. To avoid any change in the actual plant, the validation data was obtained in the same experimental arrangement but with a different initial knee joint angle. The responses of the optimised model and new experimental data showed a good agreement as shown in Fig. 8.

In the second validation process, the optimised segmental masses are validated by repeating the same optimization process for further four times. The five sets of the optimised parameters emanating from five different runs of the GA routines were examined and the results of the optimised anthropometric inertia parameters from different simulation runs exhibited acceptable repeatability with only a slight difference between each other (MSE was between 0.5 and 1.5 degrees) with small standard deviation (less than 0.01). Therefore, it can be concluded that the optimised masses obtained were valid.

\subsection{Active properties}

A new method comprising a MOGA and unconstrained MF overlap to automatically design fuzzy

Table 5

Rule base of the fuzzy model

\begin{tabular}{lccccc}
\hline Knee & \multicolumn{5}{c}{ Knee angular velocity } \\
\cline { 2 - 6 } angle & $\mathrm{NB}$ & $\mathrm{NS}$ & ZO & PS & PB \\
\hline $\mathrm{NB}$ & $(0.42) \mathrm{PB}$ & $(0.59) \mathrm{PB}$ & $(0.74) \mathrm{PB}$ & $(0.65) \mathrm{PS}$ & $(0.60) \mathrm{ZO}$ \\
$\mathrm{NS}$ & $(0.65) \mathrm{PB}$ & $(0.76) \mathrm{PB}$ & $(0.63) \mathrm{PS}$ & $(0.55) \mathrm{ZO}$ & $(0.87) \mathrm{NS}$ \\
$\mathrm{ZO}$ & $(0.49) \mathrm{PB}$ & $(0.67) \mathrm{PS}$ & $(0.87) \mathrm{ZO}$ & $(0.95) \mathrm{NS}$ & $(0.56) \mathrm{NB}$ \\
$\mathrm{PS}$ & $(0.87) \mathrm{PS}$ & $(0.78) \mathrm{ZO}$ & $(0.78) \mathrm{NS}$ & $(0.87) \mathrm{NB}$ & $(0.67) \mathrm{NB}$ \\
$\mathrm{PB}$ & $(0.85) \mathrm{ZO}$ & $(0.76) \mathrm{NS}$ & $(0.65) \mathrm{NB}$ & $(0.87) \mathrm{NB}$ & $(0.69) \mathrm{NB}$ \\
\hline
\end{tabular}



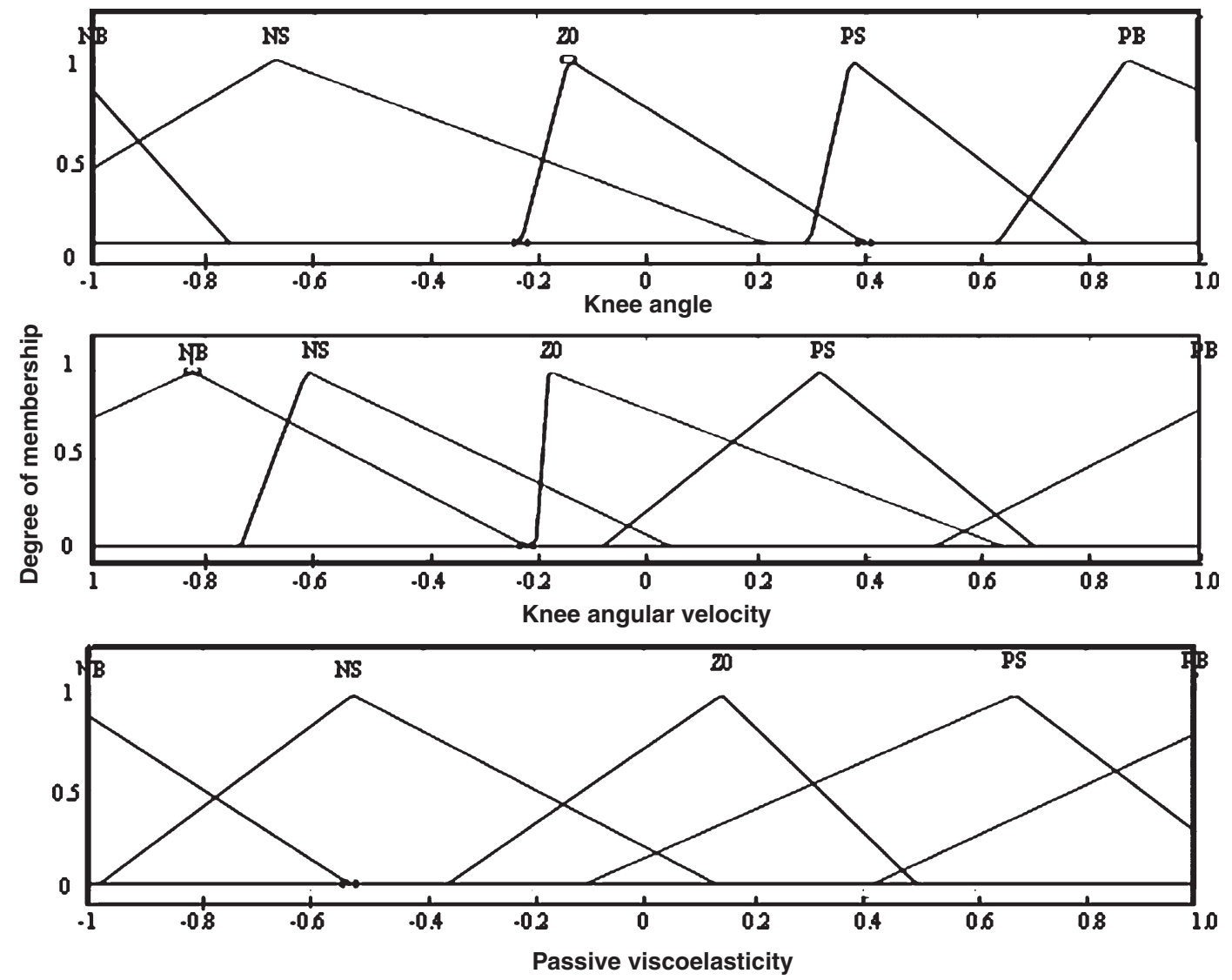

Fig. 7. Optimised membership functions of passive viscoelaslacity model.

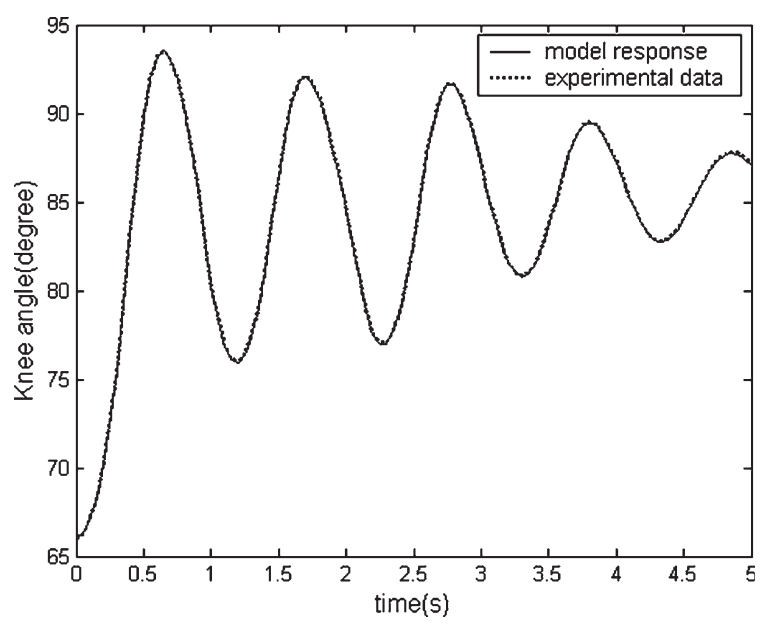

Fig. 8. Result of first validation.

systems of the active muscle properties is presented. A MOGA with 50 binary coded individuals was run for up to 200 generations to optimize the fuzzy param- eters by simultaneously minimizing both objectives. The resulting non-dominated solution front after 200 generations and table containing the corresponding parameter values are shown in Fig. 9. The best solutions achieved were with MSE $=1.88$ degrees and sum of the weighting factors of rules $=31.37$. The average weighting factor was 0.25 . The number of fuzzy rules reduced from 125 to 55 using MOGA by removing the rules with weighting factors smaller than the average value.

The response of the model was tested and the result is shown in Fig. 10. The results showed that the model parameters were estimated well and that the fit between the model and the experimental data was good. The scaling factor for the normalization and denormalization of 3 fuzzy system inputs and outputs were recorded as $\mathrm{S} 1=0.006, \mathrm{~S} 2=0.005, \mathrm{~S} 3=0.008$ and $\mathrm{S} 4=21.7$. In the fuzzy system, only 55 fuzzy rules with weighting factor more than 0.25 were used to model these active properties. Figure 11 shows the opti- 


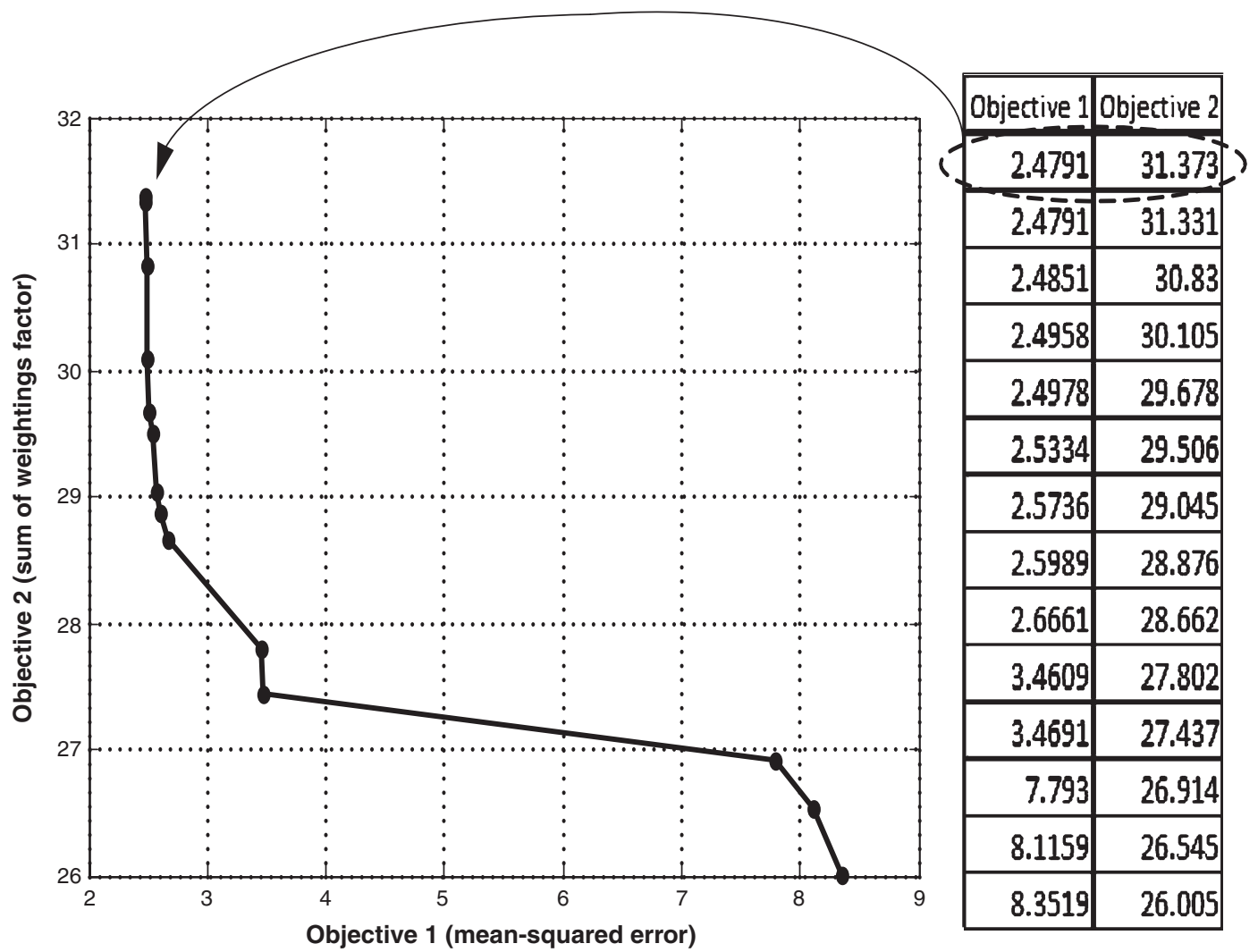

Fig. 9. MOGA optimization results.

mally shaped three inputs and one output MFs of active properties model.

The model was validated with a set of input and output data different from the modelling data. To avoid any change in the actual plant, the validation data was obtained in the same experimental arrangement but with different amplitude (slightly higher) of the

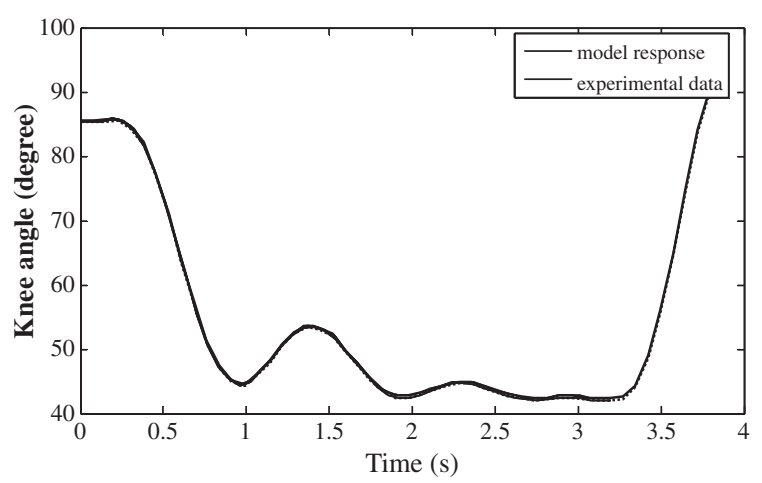

Fig. 10. The experimentally measured and simulated knee angles. stimulation pulsewidth. These input and corresponding output data were used as validation data set. The responses of the optimised model and new experimental data are shown in Fig. 12. It is seen that the experimental and model response showed good agreement with $\mathrm{MSE}=3.25$ degrees. The reduced rules fuzzy system can be used to represent the input and output non-linear behaviour of active properties of the knee joint.

\subsection{Complete knee joint model}

The active and passive properties fuzzy model thus developed needs to be integrated with optimised equations of motion to have a complete model of the knee joint as shown in Fig. 13. Equations of motion are applied to compute joint movements from joint moments taking into account gravitational and inertial effects. The purpose of modelling these properties is to use as a simulation platform of the knee joint model for control purposes. 

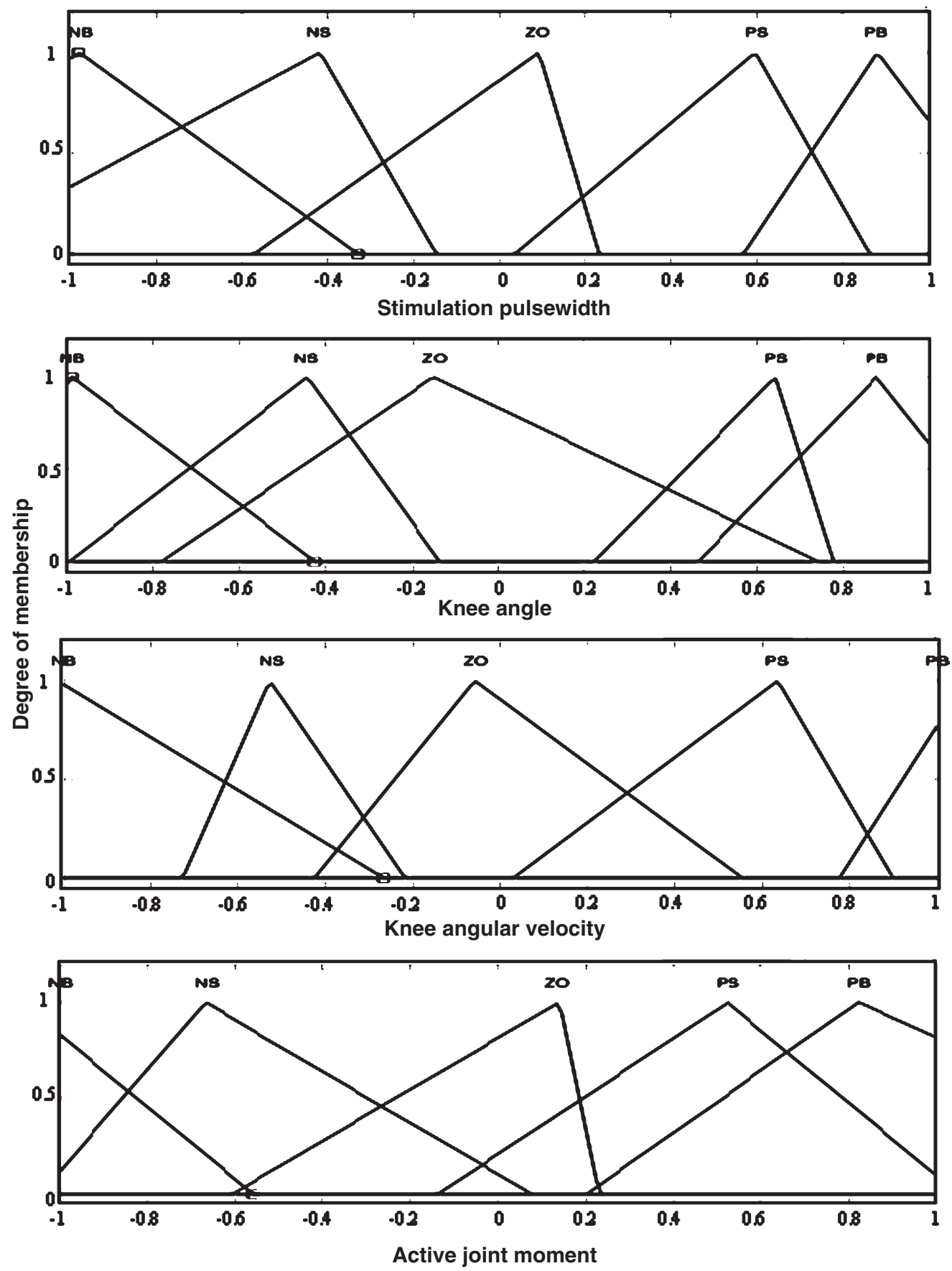

Fig. 11. Optimised membership functions of active properties model.

\section{Discussion}

Development of an effective control algorithm is reliant on accurate modelling of the system to be con- trolled. The active properties model is integrated with passive properties and optimised equation of motion to have a complete model of the knee joint. The passive properties and active properties could be difficult 


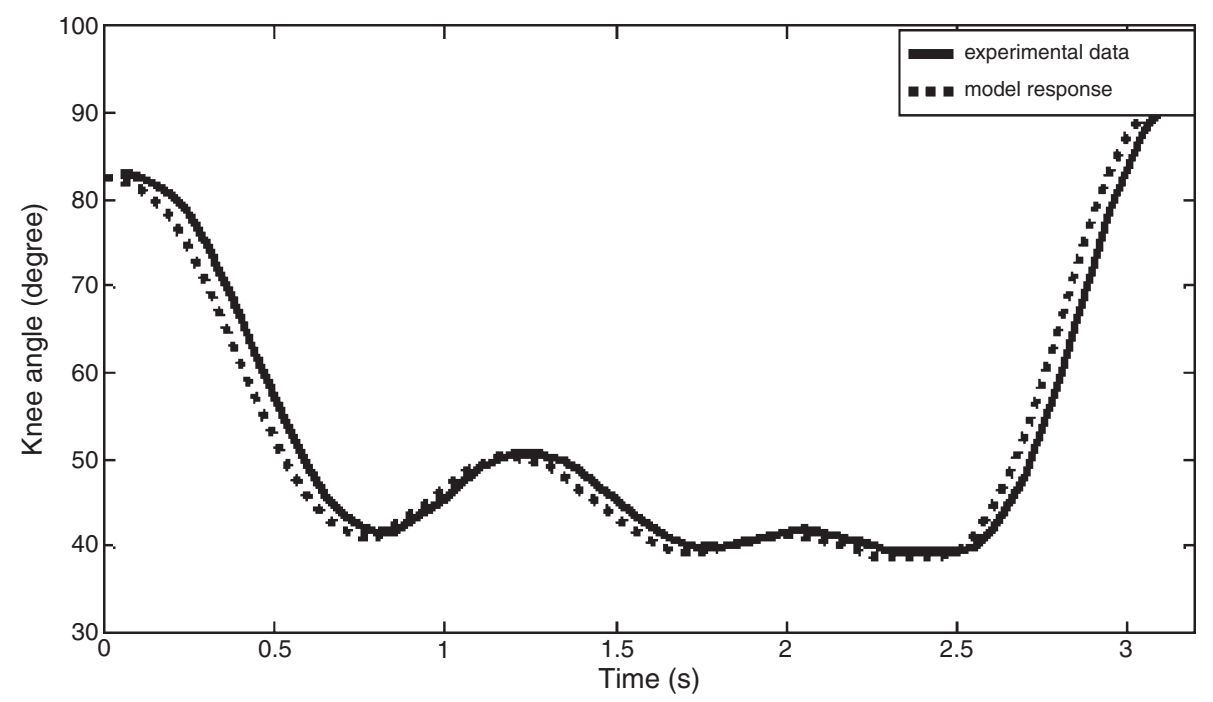

Fig. 12. Active model validation results.

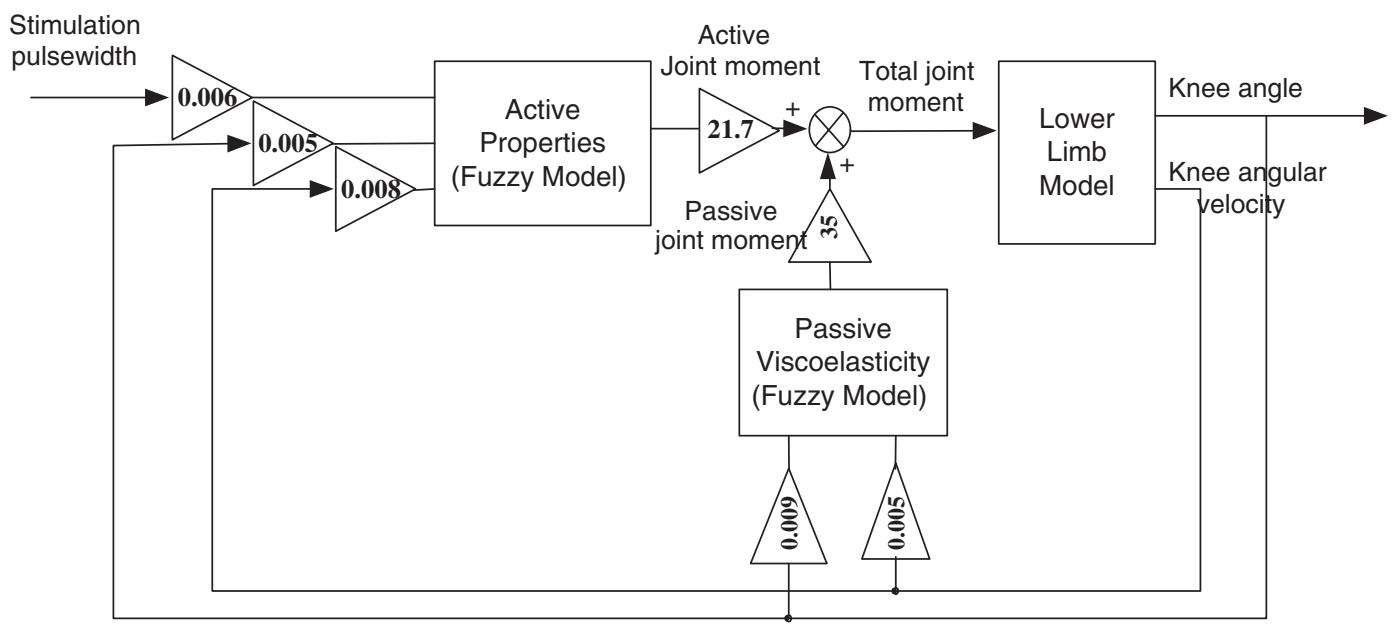

Fig. 13. Complete knee joint model.

to model due to their complexity and nonlinearity. To the authors' knowledge, this is the first time GA optimised fuzzy logic models have been systematically used for modelling of these properties. There is a primary advantage to the use of a fuzzy logic-based modelling scheme as compared to conventional mathematical methods; fuzzy logic reduces the difficulties of modelling complex systems.

With the aid of optimization techniques, a fuzzy inference system can be created that accurately characterizes dynamic behaviour of a muscle. The most important point is that GAs are intrinsically parallel, which allows them to implicitly evaluate many schema at once. GAs are particularly well-suited to solving problems where the space of all potential solutions is truly huge such as nonlinear problems [1]. Nonlinearity is the norm, where changing one component may have ripple effects on the entire system, and where multiple changes that individually are detrimental may lead to much greater improvements in fitness when combined. Therefore, fuzzy model with genetic optimization has been used to eliminate the development of complex mathematical model and help to simplify the modelling task.

Fuzzy models with more membership functions could produce better fits, but the problem with long 
computing times rapidly become more severe as the number of rules increases. In order to reduce the system's computational time, the 125 rule base designed previously are optimized and reduced to 55 rules using MOGA as explained in section 2.8. Another contribution of this study is in the use of optimisation tools to estimate the values of anthropometric inertia parameter for each subject based on pendulum test. The anthropometric inertia parameters in these equations have been estimated within given ranges using GA. This could be an easy method to estimate these parameters without going through complicated experiments since these parameters vary for each subject. Finally, such a model can be utilized as a platform for simulation of the system and development of control approaches.

\section{Conclusion}

A new approach of modelling the knee joint of an SCI patient has been presented. While the estimated models exhibited good prediction capabilities, it is comparatively less burdened with complex mathematics. The study has identified some of the potential benefits of using fuzzy logic and GA. In comparison with conventional quantitative techniques, fuzzy logic is simpler to implement as it eliminates the complicated mathematical modelling process and uses a set of fuzzy rules instead. A generic methodology has been presented that can be adopted for accurate modelling of knee joints of specific individuals with SCI. Identifying a generic and robust part of this model along with minimal set of subject-specific parameters could potentially comprise future work on the current modelling techniques. Future work may also include using this model as part of a larger musculoskeletal model of the lower limb for computer simulation studies.

\section{References}

[1] A. Marczyk, Genetic Algorithms and Evolutionary Computation, available at: http://www.talkorigins.org/faqs/genalg/ genalg.html, 2004, accessed June 2010.

[2] A. Selk Ghafari, A. Meghdari and G.R. Vossoughi, Estimation of the human lower extremity musculoskeletal conditions during backpack load carrying, Journal of Scientia Iranica 16(5) (2009), 451-462.

[3] A. Yardimci, A Survey on Use of Soft Computing Methods in Medicine, Artificial Neural Networks - ICANN 2007,
Springer Berlin/Heidelberg 2007, pp. 69-79.

[4] B. Vatnsdal, P.E. Ingvarson, D. Rafolt, V. Gudmudsdottir, S. Knutsdottir, S. Yngvason and T. Helgason, Monitoring of knee joint mechanics during electrical treatment of denervated degenerated muscle, Proceeding of the 13th Annual Conference of the IFESS, 2008.

[5] C. Fonseca and P. Fleming, Genetic algorithms for multiobjective optimization: Formulation, discussion and generalization, Genetic Algorithms, Proceeding of the Fifth International Conference, San Mateo, CA 1993, pp. 416-423.

[6] C. Karr, Applying genetics to fuzzy logic, AI Expert, pp. 38-43, March 1991. Children 6-18 years of age, Journal of Biomechanics, 41(11) (2008), 2458-24647.

[7] D. Dinakaran, S. Sampathkumar and N. Sivashanmugam, An experimental investigation on monitoring of crater wear in turning using ultrasonic technique, International Journal of Machine Tools and Manufacture 1 (2009), 109-120.

[8] D.E. Goldberg, Genetic Algorithms in Search, Optimization and Machine Learning, Reading, Addison-Wesley, MA, 1989.

[9] D.A. Winter, Biomechanics and Motor Control of Human Movement. 2nd ed., Wiley-Interscience, New York, 1990.

[10] G.H. Shue and P.E. Crago, Muscle-tendon model with length history dependent activation-velocity coupling, Annual Biomedical Engineering 26(3) (1998), 369-380.

[11] G.I. Zahalak and S.P. Ma, Muscle activation and contraction: Constitutive relations based directly on cross-bridge kinetics, Journal Biomechanical Engineering 112(1) (1990), 5262.

[12] H. Ishibuchi, K. Nozaki, N. Yamamoto and H. Tanaka, Selecting fuzzy if-then rules for classification problems using genetic algorithms, IEEE Transactions on Fuzzy Systems, 3(3) (1995), 260-270.

[13] H.N. Teodorescu, A. Kandel and L.C. Jain, Fuzzy and NeuroFuzzy Systems in Medicine, CRC Press, LLC, Boca Raton, Florida, 1999.

[14] J. Bobet and R.B. Stein, A simple model of force generation by skeletal muscle during dynamic isometric contractions, IEEE Trans Biomed Eng 45(8) (1998), 1010-1016.

[15] J. Ding, A.S. Wexler and S.A. Binder-Macleod, A mathematical model that predicts the force-frequency relationship of human skeletal muscle, Muscle Nerve 26(4) (2002), 477485.

[16] M.B.I. Reaz, M.S. Hussain and F. Mohd-Yasin, Techniques of EMG Signal Analysis: Detection, Processing, Classification and Application, Biological Procedures Online, 8(1) (2006), 11-35.

[17] M. Ferrarin and A. Pedotti, The relationship between electrical stimulus and joint torque: a dynamic model, IEEE Transactions on Rehabilitation Engineering 8(3) (2000), 342-352.

[18] M. Gen and R. Cheng, Genetic Algorithm and Engineering Optimization, New York, Chichester, Wiley-Intersection, 2000.

[19] M.K. Lebiedowska, Dynamic properties of human limb segments, in: International Encyclopedia of Ergonomics and Human Factors, C.R.C. Press, 1 2006, pp. 315-319.

[20] M.K. Lebiedowska, The kinematic consequences of invariant dynamics, in: Use of Genetic Algorithms to Solve Biomedical Problems, M. Levin, Ed, M.D. Computing, vol. 12, no. 3, 1995, pp. 193-198. 
[21] M.S. Valle, A. Casabona, R. Sgarlata and R. Garozzo, The pendulum test as a tool to evaluate passive knee stiffness and viscosity of patients with rheumatoid arthritis, BMC Мusculoskeletal Disorder 7 (2006), 89-100.

[22] M.S. Huq, Analysis and Control of Hybrid Orthosis in Therapeutic Treadmill Locomotion for Paraplegia, $\mathrm{PhD}$ Thesis, The University of Sheffield, Sheffield, UK, 2009.

[23] R. Massoud, Intelligent Control Techniques for Spring Assisted FES-Cycling, $\mathrm{PhD}$ Thesis, The University of Sheffield, Sheffield, UK, 2007.

[24] R. Perumal, A.S. Wexler and S.A. Binder-Macleod, Development of a mathematical model for predicting electrically elicited quadriceps femoris muscle forces during isovelocity knee joint motion, Journal Neuroengineering Rehabilitation 5 (2008), 33.

[25] R. Riener and T. Edrich, Significance of passive elastic joint moments in FES, Proceedings of the 2nd Conference of IFESS, 1997, pp. 103-106.
[26] R. Riener and T. Fuhr, Patient-driven control of FESsupported standing up: A simulation study, IEEE Transactions on Rehabilitation Engineering 6(2) (1998), 113-124.

[27] R. Wartenberg, Pendulousness of the leg as a diagnostic test, Neurology 1 (1951), 18-24.

[28] T.C. Chin and X.M. Qi, Genetic Algorithms for learning the rule base of fuzzy logic controller, Fuzzy Sets and Systems 97 (1998), 1.

[29] T.R. Kane and D.A. Levinson, Dynamics: Theory and Applications, McGraw Hill, New York, 1985.

[30] V.Zatsiorsky and V. Seluyanov, The Mass and Inertia Characteristics of the Human Body, Biomechanics VIII-B, University Park Press 1983, pp. 1152-1159.

[31] Y. Jin, Fuzzy modeling of high-dimensional systems: complexity reduction and interpretability improvement, IEEE Trans on Fuzzy Systems 8 (2000), 212-221. 

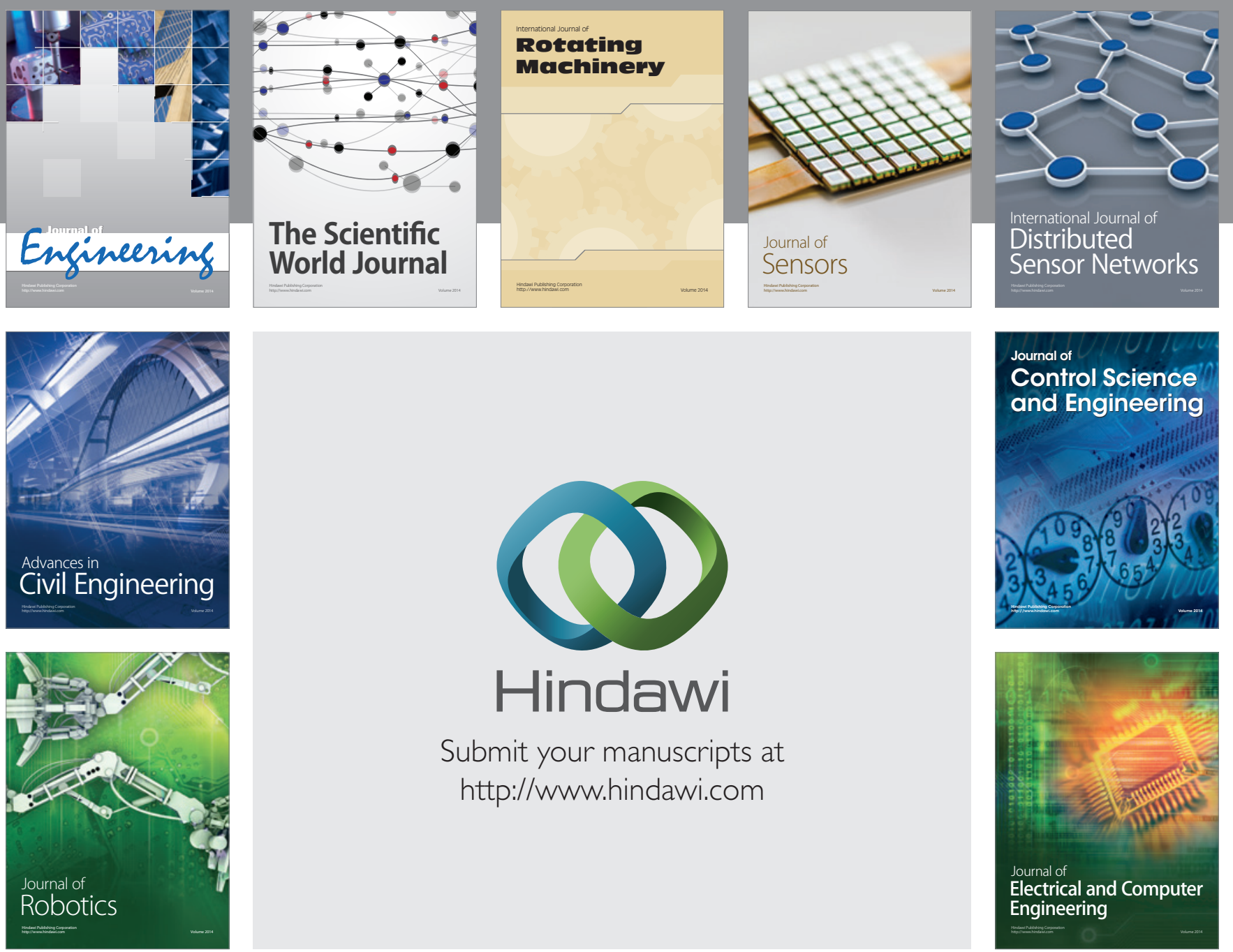

Submit your manuscripts at

http://www.hindawi.com
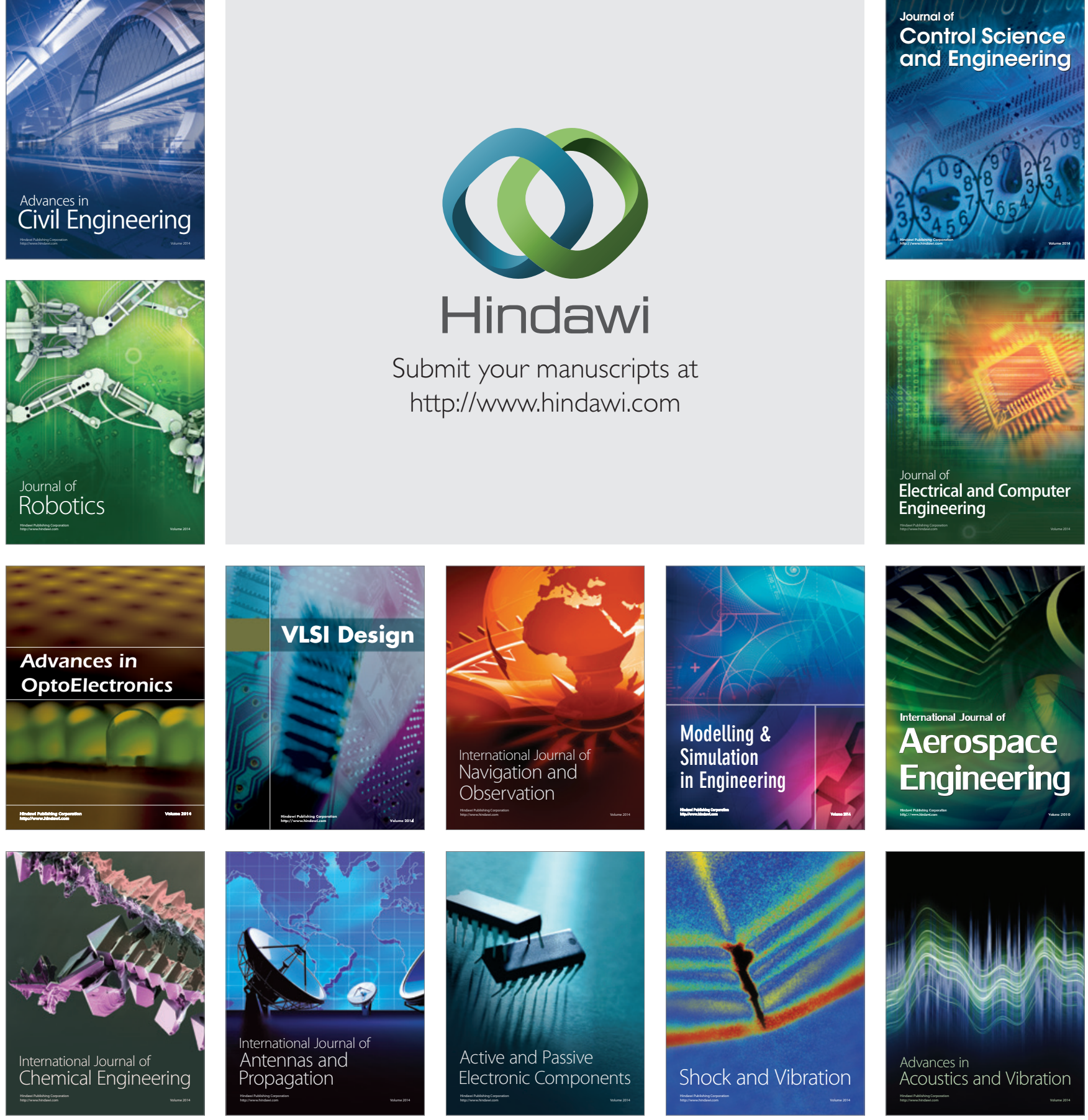